\title{
M-Ary Spread Spectrum Code Division Multiple Access Underwater Acoustic Communication*
}

\author{
Yu Yang ${ }^{1)}$ Duan Min ${ }^{1)}$ \\ ${ }^{1}$ School of Traffic and Transportation, Xuchang University, Xuchang, 461000
}

Keywords: underwater acoustic communication, spread spectrum, M-ary, CDMA

\begin{abstract}
M-ary spread spectrum telemetry is employed to overcome the constraints imposed by spreading factor in data rate. In this paper, M-ary spread spectrum code division multiple access (CDMA) underwater acoustic (UWA) communication is proposed to improve data rate of conventional CDMA, and the data rate enhancement of M-ary CDMA is investigated. The performance of the conventional and proposed schemes over sloping sea channel with additive white Gaussian noise has been evaluated. The effect of multiple user interference (MUI) is investigated through analytical expressions and simulation to exhibit the characteristics of the proposed scheme. Numerical results and comparison of the two methods in terms of equal data rate are also given.
\end{abstract}

\section{Introduction}

Underwater channel is a very complex medium for acoustic communications because of noise, attenuation, multi-path propagation and Doppler shift ${ }^{[1]}$. In UWA channel, attenuation of signal enhances with the increasing of range and frequency, while power spectrum density (PSD) of ambient noise decreases with the increasing of frequency in available bandwidth. As a result, there is an optimal carrier frequency for different range, and the optimal frequency decreases with the increasing of range. Bandwidth available for UWA channel is also relatively limited as compared to other media. In practice, the available bandwidth is even smaller than what is theoretically predicted from the consideration for environment factors ${ }^{[2]}$.

The need to sense the underwater world drives the development of underwater sensor networks. Applications of underwater networks fall into three main categories, which are broadly classified as scientific, industrial and military applications ${ }^{[3]}$. In a networked environment, CDMA is a preferred choice for giving many users simultaneous access to the medium and hence is adopted on account of its relaxed synchronization requirement and efficient bandwidth utilization ${ }^{[4]}$.

\section{Basics of M-ary CDMA}

M-ary CDMA is introduced as the communication scheme in this paper in that it offers higher data rate as compared to conventional scheme. In this paper we adopt Gold sequences due to their good cross correlation properties. If we define $r$ as the order of sequences, $K$ is number of users, and $2^{r}+1$ is the available size of code sets, then the code size distributed to each user is $\left\lfloor\frac{2^{r}+1}{\mathrm{~K}}\right\rfloor$, here \lfloor\rfloor represents floor of the fraction by rounding. If the conventional CDMA data rate of each user is defined as basic rate in the paper hereafter. Then, the data rate of M-ary CDMA is $\log _{2}\left\lfloor\frac{2^{r}+1}{K}\right\rfloor$ times as high as basic rate.

The block diagram of the proposed scheme is as follow: 


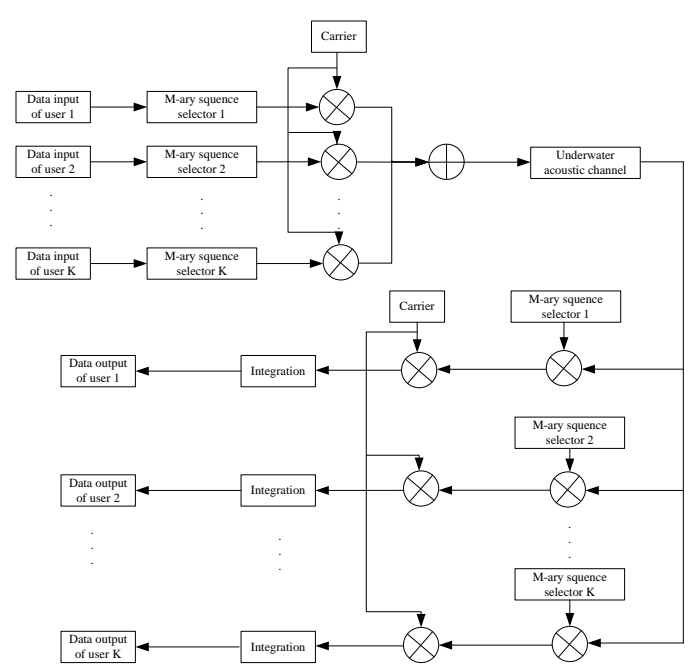

Fig 1. M-ary CDMA block diagram

First of all, each user selects one of the Gold sequences of code sets according to its input information. These sequences are then, modulated by a carrier. After modulation, modulated sequences from each user are added and transmitted signal is formed. The signal of each user can be expressed as

$$
\mathrm{s}_{i}(t)=A_{i} c_{i, j}(t) \cos \left(\omega_{c} t+\varphi\right)
$$

Thus, where $\mathrm{s}_{i}(t)$ denotes the transmitted signal.

After the contamination of noise and UWA channel, the received signal can be written as

$$
\mathrm{r}_{i}(t)=\sum_{i=1}^{K} \sum_{l=1}^{L} A_{i, l} c_{i, j}\left(t+\tau_{l}\right) \cos \left(\omega_{r} t+\varphi_{l}\right)+n(t)
$$

Where $\tau_{l}$ denotes delay of different paths, $\omega_{r}$ is the received angular frequency.

Demodulated signal of user 1 is given below as an example, $c_{1, m}(t)$ is the local Gold sequence of user $1, \cos \left(\omega_{r} t+\varphi_{1}\right)$ denotes local carrier. It is assumed that the synchronization of transmitted and received code sequences has been achieved.

$$
\begin{aligned}
& \mathrm{V}_{1, m}(t)=\frac{1}{2} A_{1} \int_{\tau_{1}}^{T+\tau_{1}} c_{1, m}\left(t+\tau_{1}\right) c_{1, j}\left(t+\tau_{1}\right) d t \\
& \quad+\frac{1}{2} \sum_{i=2}^{K} A_{i} \int_{\tau_{1}}^{T+\tau_{1}} c_{1, m}\left(t+\tau_{1}\right) c_{i, j}\left(t+\tau_{l}\right) d t \\
& \quad+\int_{\tau_{1}}^{T+\tau_{1}} n(t) c_{1, m}\left(t+\tau_{1}\right) \cos \left(\omega_{r} t+\varphi_{1}\right) d t+I_{1, m}(t)
\end{aligned}
$$

The first term of expression (3) is output of the desired user. The second term can be regarded as interference of other users to the desired one. The third part is the contribution of noise, and the forth part is the effect of multi-path propagation. The last part of the mentioned expression above is given as

$$
I_{1, m}(t)=\frac{1}{2} \sum_{i=1}^{K} \sum_{l=1}^{L} A_{i, l} \int_{\tau_{1}}^{T+\tau_{1}} c_{1, m}\left(t+\tau_{1}\right) c_{i, j}\left(t+\tau_{l}\right) \cos \left[\omega_{c}\left(\tau_{1}-\tau_{l}\right)\right] d t
$$

The expression (4) is interference of multi-path propagation caused by all users. $A_{i, l}$ and $\tau_{i, l}$ denote amplitude and delay of user $i$ and path $l$.

\section{Numerical results}

In this section we present the comparison between conventional CDMA and M-ary CDMA communication scheme. The data rate obtained using the above mentioned methods according to different number of users can be expressed as multiple of basic rate. The code length is 63 for both two methods. The relationship between communication data rate and number of users is shown in 
figure 2 for both CDMA and M-ary CDMA schemes.
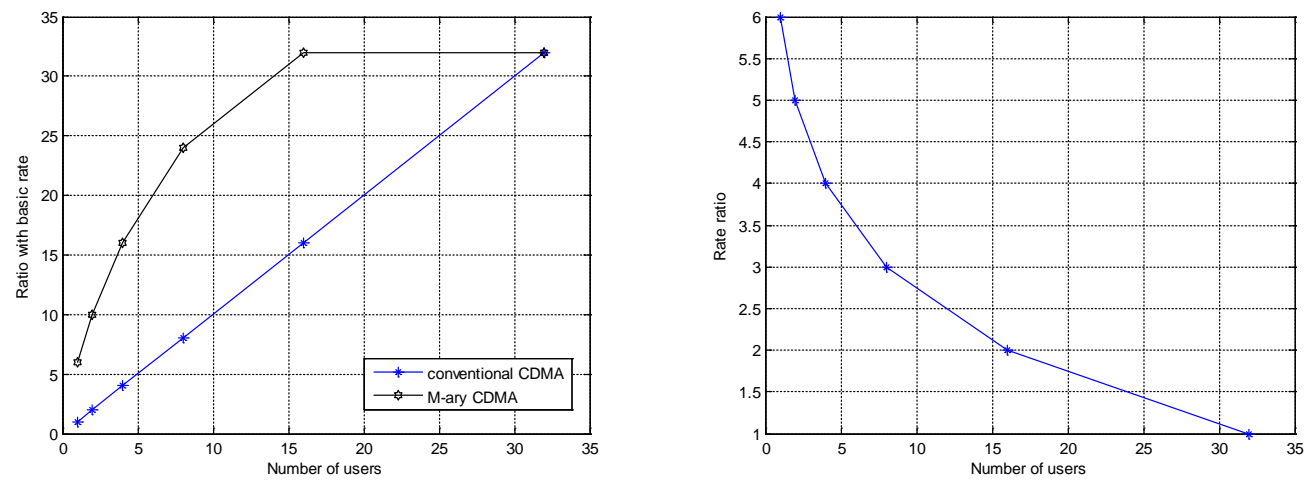

Fig. 2 The comparison of data rates

Fig. 3 The rate ratio between M-ary CDMA and conventional CDMA

It is demonstrated that the data rate of M-ary CDMA is higher than that of conventional CDMA. The ratio of the rates achieved by conventional CDMA and M-ary CDMA is given in figure 3. The difference in the rates decreases with the increasing of number of users. It is revealed that the data rates of two communication methods will converge at a certain number of users and the advantage of M-ary CDMA will reduce as number of users increases from figure 3.

The simulation has been performed using an underwater channel response near Ormara which is an important seaport of Pakistan. The location belongs to continental slice of ocean cross-section in which the depth increases from $10 \mathrm{~m}$ to $722 \mathrm{~m}$. The range is $20 \mathrm{~km}$. The depths of transmitter and the receiver are $5 \mathrm{~m}$ and $400 \mathrm{~m}$, respectively. The channel impulse response (CIR) is

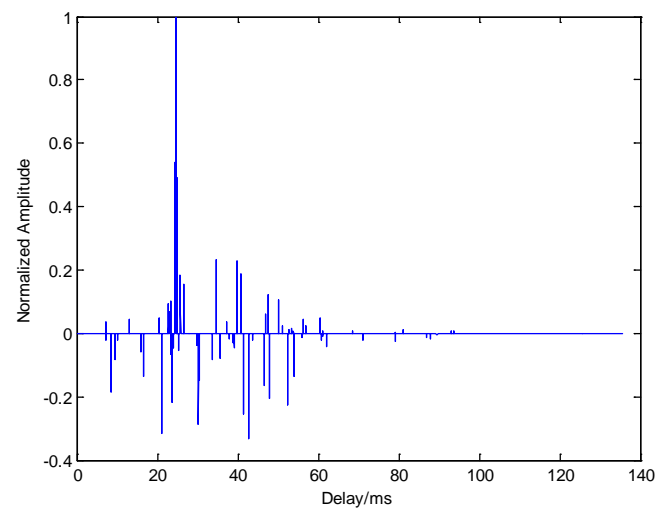

Fig. 4 Sloping sea CIR

It can be seen that multi-path delay is on the order of tens of milliseconds and the CIR is a non-minimum phase system in that longer distance paths travel at higher speeds. This channel is called UWA channel for brief.

Figure 5 depicts the comparison of bit error rate (BER) curves between synchronous conventional CDMA and M-ary CDMA over AWGN channel. The simulation adopts $8 \mathrm{kHz}$ as carrier frequency with $4 \mathrm{kHz}$ bandwidth and $48 \mathrm{kHz}$ sampling frequency. For conventional CDMA we have used m sequences with code length 63 , while M-ary scheme adopts Gold sequences of the same length. The conventional CDMA method utilizes DSSS. 


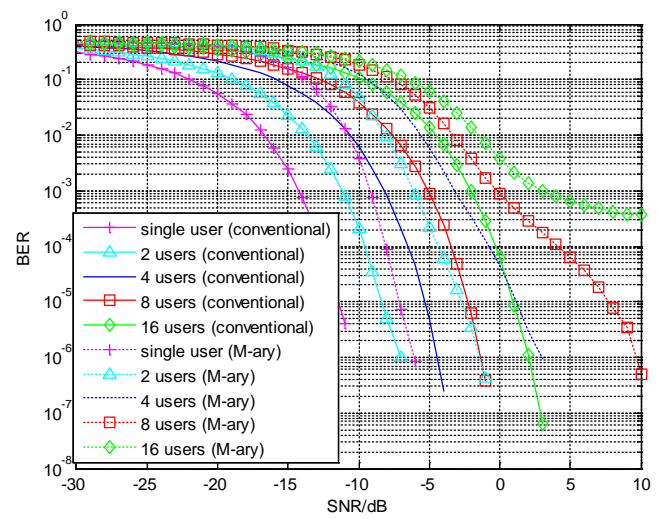

Fig. 5 BER comparison between two schemes over AWGN channel

Some basic characteristics of the two schemes are shown in figure 5 where performance of the schemes is made known for different number of users. Here we can see curves from single user to 16 users utilize the bandwidth at the same time. It can be concluded that noise tolerance ability will decline as the number of users increases.

The simulation parameters are as same as which have been used for the previous case in figure 5 . The comparison of BER for the UWA channel is as given in figure 6.

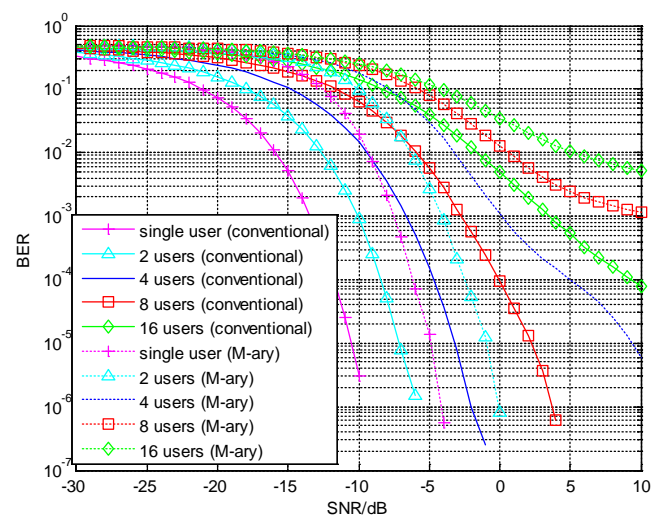

Fig. 6 BER comparison between two methods over sloping sea channel

The performance of the schemes in this channel is not as good as in AWGN channel, and it deteriorates when the number of users increases. The BER curves do not decrease beyond a certain error rate even if we go on increasing the SNR for the cases of 8 and 16 users. It can be inferred that the fading channel impacts the robustness of system especially for the high number of users. This can be attributed to the interference of second terms in expression 3 and 4 which enhance as the multi-path complexity increases.

\section{Conclusion}

In this paper, M-ary CDMA UWA communication system is proposed to improve the communication data rate of conventional CDMA. In conventional CDMA, the data rate can be increased at the cost of decreasing the spreading gain, and the low spreading gain deteriorates the performance of CDMA. So a trade off between data rate and performance exists in the conventional method of multiple user access communication scheme. M-ary telemetry has been used to overcome the problem of this compromise in spreading factor and communication rate.

\section{Reference:}

[1] Kilfoyle D B, Baggeroer A B. The state of the art in underwater acoustic telemetry. IEEE J. Ocean. Eng. 2000, 25(1): 4-27

[2] Stojanovic M, Preisig J. Underwater acoustic communication channels: propagation models and statistical characterization. IEEE Commun. Mag. 2009, 47(1): 84-89 
[3] Heidemann J, Stojanovic M, Zorzi M. Underwater Sensor Networks: Applications, Advances, and Challenges. Philosophical Transactions of the Royal Society-A 2012, 370(1958): 158-175

[4] Rice J, Green D. Underwater acoustic communications and networks for the US Navy's Seaweb program. The second international conference on sensor technologies and applications. Washington D C, USA. 2008: 715-722. 